\title{
The Development of Cognition and Application of Bio-inspired Design
}

\author{
Jining $\mathrm{Lu}^{1,2, a}$, Bochu $\mathrm{Xu}^{1, \mathrm{~b}}$ and Feng $\mathrm{We}^{\mathrm{i}^{1, \mathrm{c}}}$ \\ ${ }^{1}$ School of Architecture and Design, Southwest Jiaotong University, Chengdu 610031, China; \\ 2 School of Art, Chongqing Technology and Business University, Chongqing 400067, China. \\ alujining_cq@qq.com, b273786680@qq.com, cwei.feng58@163.com
}

\begin{abstract}
Keywords: Bio-inspired Design, Design Application, Design Cognition, Design Development.
\end{abstract}
\begin{abstract}
Bio-inspired design has always been a design hotspot and its research development allows us to understand its characteristics and methods more clearly. According to our knowledge about it and application degree, the development process of bio-inspired design was divided into four different stages: the uncivilized stage, the initial stage, the intermediate stage and the advanced stage, which pointed out the different levels of people's cognition and application and set forth its development characteristics that from merely imitation to multi-disciplinary integrated application. The future of bio-inspired design was also forecasted.
\end{abstract}

\section{Introduction}

Biomimicry, which also be called as "Bionics" or "Biomimetic", is a science to design new technology systems through imitating the structure, physical and functional principles of biological systems [1-3], and its application in the design field is bio-inspired design [4]. After hundreds of millions of years' evolution, various organisms have adapted to survival their natural environment, and those abilities attract people to imagine and imitate, which make bio-inspired design being an enduring design trends.

When people do a bio-inspired design, first of all, they must have a scientific and comprehensive understanding on natural phenomenon or biological function. Besides of that, they also should have the ability to apply those principles to various man-made products. Hence according to people's understanding on bio-inspired design and its application extent, it could be divided into the following development stages.

\section{The Uncivilized Stage of Bio-inspired Design}

In ancient times, since harsh forces of nature were huge threats to human's living. Capability and strength became the main factor of people's survival and development. Primitive man's worship of nature is actually a worship of power, and they are yearning to it. Strong body, rapid speed, and extraordinarily suitable forms are all favorable conditions for self-preservation and to defeat opponents. Therefore, the essence of these phenomena is great power, and this power becomes the object for people to fear, to worship and to imitate.

Being limited to their cognitive ability and technical means, primitive man simply thought that those biological organs were the source of magical powers. In order to obtain these magical powers, they put some organs in their own body, such as wrapping their bodies with fur peeled off from animals to get warm. Another example is, in the fight with the beast, they found that animal's sharp teeth and claws were effective tools, hence they remove the claws and teeth from those animals, and add branches to increase the length. That was the original spear. Some myths and legends also reflected such cognition, such as the Greek mythology about Daedalus wings.

At this stage, their understanding of biological organs is chaotic, cannot distinguish function from shape, structure, material, etc., and to find out the decisive factor. Therefore, they can only imitate the organism as a whole, and take their original organs as tools. 


\section{The Initial Stage of Bio-inspired Design}

With the accumulation of knowledge, people acquired some knowledge about those shapes that easy to be observed, and be able to distinguish function from the biological organ itself, and to imitate the biological appearance and external structure.

As for the original spear mentioned above, people found that the sharp head and the hard material are the keys to kill a prey effectively, so they began to point the tip of the tree branches directly, or tie a sharp stone whose shape is exactly same as animal's claws or teeth at the stick's front part, and finally they chosen tough metal to make these weapons. Four thousand years BC, our ancestors saw the bitter fleabane spinning in the wind and hence invented the wheel. In Chinese legend, Lu Ban was inspired by the sharp teeth of grass leaves which hurt him when he grabbed them and hence invented the saw. A Greek mythology tells that it is Talos who was inspired by the shape of jawbone or spine of fishes and invented the saw.

At this stage, limited to human knowledge accumulation, cognitive ability and technical means, the imitation of nature is mainly concentrated in the external form and explicit structure. A bio-inspired design mainly depended on choosing appropriate material, and to produce the appropriate shape.

The imitation of living things is not purely for practical purposes, as well as for aesthetic factors. Maslow notes that people need beauty is just as a person's diet needs calcium, and beauty can help people become healthier [5]. In the long-term contact with surrounding creatures, after sawing their exuberant vitality and their ability to adapt to the environment, people would automatically love these geniuses of nature, and describe them on human bodies and tools.

The primitive totem worship of creatures, the cognition about biotic forms' effects, and the aesthetic experience associated with these visual images, all three factors contribute to the next stage of bio-inspired design. That is, when imitating those organisms, people not only wanted to achieve a certain function, but also wanted to cause some kind of aesthetic experience.

In addition, in the long-term evolutionary process, people gradually give those biotic forms various symbolic meanings, such as ferocity makes lion a symbol of royal authority, soft fur and gentle temperament makes rabbit a symbol of gentle and soft. People have also begun to consciously use these biotic forms to convey the symbolic meaning they have been given, such as the chairs of Pharaohs of Egypt, decorated with lion's heads and claws (Fig. 1). The same situation occurs to dragon chair in China.

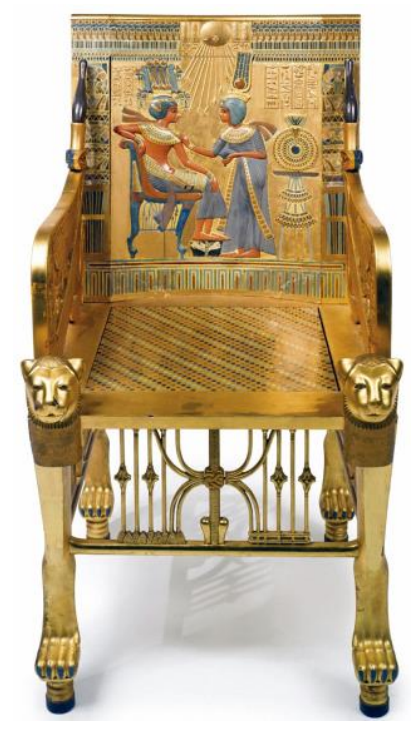

Fig. 1 Egyptian Pharaoh's throne

\section{The Intermediate Stage of Bio-inspired Design}

At the intermediate stage of bio-inspired design, since people had had an improved understanding to nature, they could distinguish the major features of imitated objects from minor features, and could 
accomplish abstract biomimicry by grasping major features and neglecting minor ones. This kind of abstraction is quite different from the original abstraction of the buffalo painted on the walls by primitive man. Primitive abstraction is due to their poor cognitive ability and performance techniques. They wanted to express concrete images but they couldn't, so we can say that their abstraction is a passive one. Modern abstraction is formed on the base of people's improved cognition about nature and matured performance techniques. This abstract form is started from the specific things, endured repeated scrutiny, being removed all the trivial details and get the general conclusion. It is a kind of active abstraction. This abstraction is a reflection of the unique essence of things, is so-called "from the concrete and beyond the concrete". A good example is those designs appeared in Art Nouveau (Fig. 2). A representative imagery of the Art Nouveau style is the organized, intertwined and dynamic curves, which are abstracted from the natural vegetation, with flowing shapes and meandering lines, full of inner vitality, which conveys the rhythms of movement and the growth of the organism, embodies the endless process of creation under the surface of living things. These patterns have been used in furniture, and have become a symbol and metaphor of life.

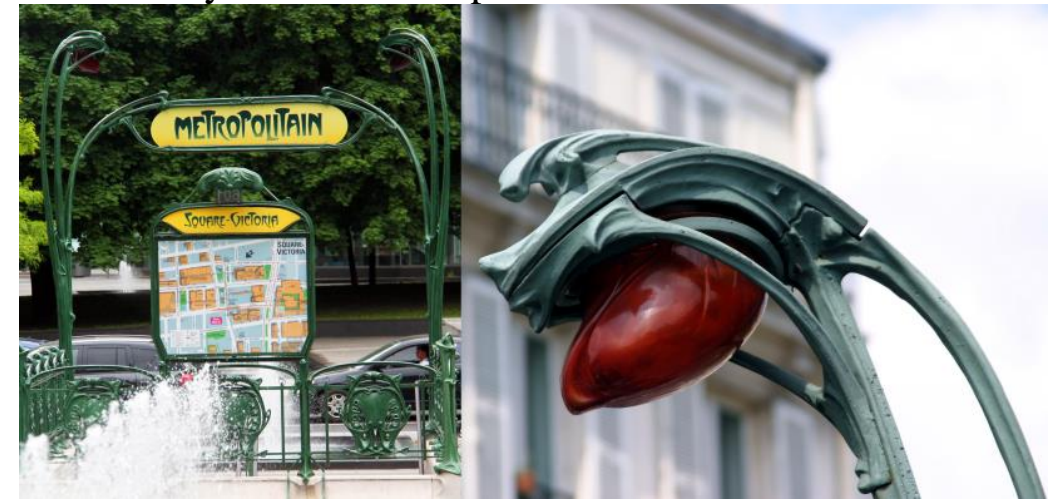

Fig. 2 the Paris subway entrance designed by Guimarde

As for the symbolic meaning of biomimetic styling, it becomes more meaningful and systematic at this stage. For example, the European Medieval Gothic architecture and later the Gothic furniture, often took those images like pointed arch and pinnacle. In those images vertical lines are deliberately stressed [6]. Almost all these kinds of biomimetic patterns contain some Christian symbols. For example, trefoil symbolizes the Trinity of the Father, the Son and the Holy Spirit; quatrefoil symbolizes the Four Gospels; cinquefoil symbolizes the five Apostles; a white pigeon represents the Holy Spirit; a white lily signifies purity and divinity of the Virgin Mary (see Fig. 3).

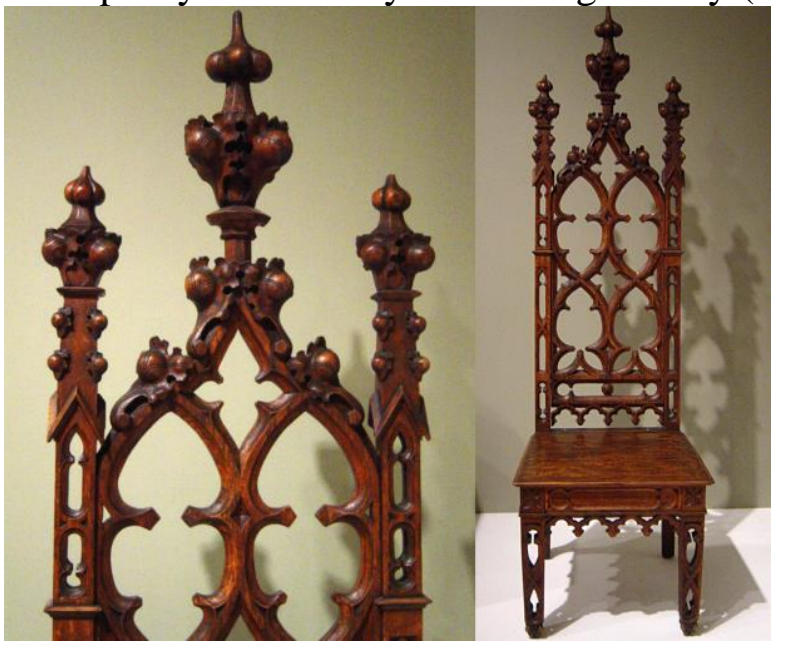

Fig. 3 Gothic furniture decoration

Those highly abstract forms, with characteristics of illegibility and polysemy, also cause different understanding to different cognitive subjects, that is, "the benevolent see benevolence and the wise see wisdom". A typical example is the La Chapelle de Ronchamp designed by Corbusier, which takes an unusual form and can trigger people's associations, such as a folded hand, a ship, a duck floating on the water, or a hat (fig. 4) .This phenomenon means the abstract art have been developed to a higher level, 
and designers began to consciously apply a more vague appearance to guide people to form a variety of cognitions and speculations, in order to convey some comprehensive, not so precise meanings, or to cause some unspeakable emotions.
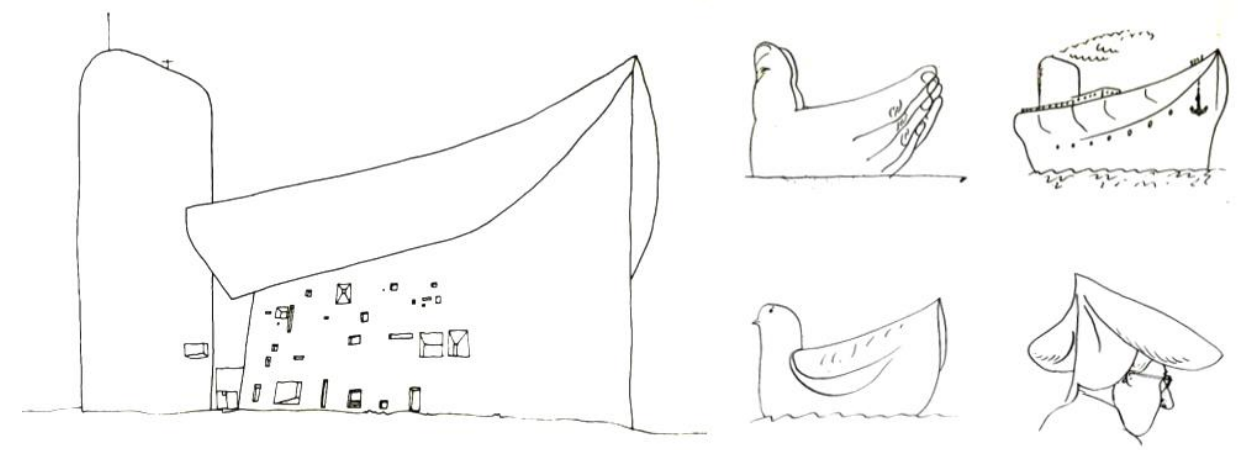

Fig. 4 different understandings on La Chapelle de Ronchamp

\section{The Advanced Stage of Bio-inspired Design}

As the knowledge system becomes more complex and systematic, the understanding to nature and to design tasks faced by human being themselves becomes more and more in-depth, each important bio-inspired design would do its analysis and research from mechanics, electronics, optics, thermology, biology, art, psychology, sociology and other aspects, referred to many disciplines mentioned before, and becomes a comprehensive application of interdisciplinary.

The artificial dolphin skin research process directly reflects the characteristics of this stage. Dolphins can travel up to $70 \mathrm{~km} / \mathrm{h}$. Since it hardly produces the kind of turbulence which caused by man-made submersible, it bears minimal water drag. Max Krame, a scientist, noticed it and began to study dolphins. He used plastic, steel and rubber to make a model almost exactly the same size as a real dolphin, a radio to control the model's activities, and ensure that the power of model was the same as that of true dolphins. The result is that the model travels at a speed of only $3.6 \mathrm{~km} / \mathrm{h}$ which is much slower than a true dolphin's, and it has far less energy utilization ratio than live dolphins. After extensive research, Kramer found that dolphins not only have an ideal streamlined body shape, but also special skin structures to reduce the vibration amplitude. A hydrodynamic study shows that the generation of turbulence near an object surface is related to its vibration. The structure of dolphin's skin allows it to transform with changes of flow velocity and water drag, so as to dampen vibration and prevent turbulence effectively. This phenomenon is called "self-adaptive". Meanwhile, the dolphin's body fluids secreted from skin will increases with increasing water pressure. This body fluid is hydrophobic, it makes water that contacted dolphins' body to form some small droplets, like the ball in the bearing, to minimize the frictional drag. Besides, when the dolphin moves at a high speed, its subcutaneous muscles will begin to take a wave-type contraction, to eliminate vortexes and turbulences caused by high-speed movement. Based on this understanding, Kramer completed the lamiflo (i.e. artificial dolphin leather) by bio-inspired design, which was used in various environments where there is a need to reduce the fluid drag [7].

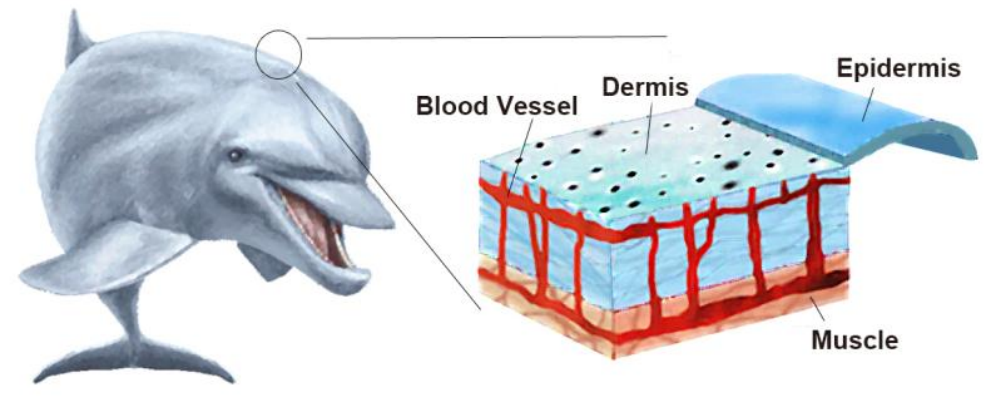

Fig. 5 Microstructure of dolphin skin 
The research process on dolphin skin clearly shows that it is necessary to have a comprehensive system research on multiple disciplines, including biology, mechanics, electronics, materials science, morphology, and fluid mechanics, to understand the principles of biological organs or natural structures and their operating mechanisms and hence get ideal results. The pattern of systematical research natural resource and apply biomimicry to multi-area, is a sign of the advanced stage of bio-inspired design. At this stage, through the study of shape, structure, function, energy conversion, information transmission and other excellent features of the biological system, we can apply them to technical systems, natural systems or social systems. By this kind of bio-inspired design, we can improve the existing engineering equipment, natural environment or social organization structure, and create new technical skills, automation devices, special technical components, etc. At the same time, bio-inspired design provides a new direction for the development of modern design and act as a communication "link" between human society and the nature.

\section{Future Prospects of Bio-inspired Design}

From the existing bio-inspired design development process, its further development should be to cultivate biotic tissues or organs which could be adapted to different needs through the utilization of genetic technology combination, or through the use of nanotechnology or some more advanced technologies to generate ultra-microstructure material on atom, medium proton or even lower levels of materials, so that humans can be free to get materials or devices made by different combinations of biological functions. Similar to current integrated circuits, these designs and productions will probably need specialized production lines to perform. At this stage, the bio-inspired design will totally shift its way from imitation to the creation, the humans will be free to design new structures, organisms or materials according to the characteristics of the different micro-structures. At that time, the humans' understanding and application of bio-inspired design will reach an unprecedented height and the industrial design will also enter a new era.

\section{References}

[1]. Benyus J M. Biomimicry: innovation inspired by nature. William Morrow, 1997, p. 2.

[2]. Coinau Y, Kresling B. Bionics and design: witnesses to the evolution of this approach. Temes De Disseny Disseny CommunicAsia Culture. (1994), No. 11, p. 42-51.

[3]. Vincent J F V. Biomimetics--a review. Proceedings of the Institution of Mechanical Engineers, Part H: Journal of Engineering in Medicine. Vol. 233 (2009) No. 8, p. 919-939.

[4]. Helms M, Vattam S S, Goel A K. Biologically Inspired Design: Process and Products. Design Studies. Vol. 30 (2009) No. 5, p. 606-622.

[5]. Maslow A. Humanistic Philosophy of Maslow. Jiuzhou Press, 2003, p. 64.

[6]. He Renke. A history of Industrial Design. Beijing Institute of Technology Press, 1991, p. 35.

[7]. Wang Shurong. Inspirations of Nature. Shanghai Scientific and Technical Publishers, 1978, p. 173-177. 